11,10

\title{
Фазовый переход металл-диэлектрик в тонких пленках диоксида ванадия, легированного железом
}

\author{
() В.Н. Андреев, В.А. Климов
}

Физико-технический институт им. А.Ф. Иофрфе РАН, Санкт-Петербург, Россия

E-mail: vn.andreev.solid@mail.ioffe.ru

(Поступила в Редакцию 22 мая 2018 г.)

Проведены исследования электропроводности поликристаллических пленок $\mathrm{V}_{(1-x)} \mathrm{Fe}_{x} \mathrm{O}_{2}$ в широком интервале температур, охватывающем область как металлической, так и диэлектрической фазы. Показано, что при увеличении концентрации железа фазовый переход металл-диэлектрик смещается в сторону низких температур, тогда как температурная область перехода в легированных образцах испытывает дополнительное уширение по сравнению с чистым $\mathrm{VO}_{2}$. Для объяснения температурной зависимости электропроводности диэлектрической фазы $\mathrm{V}_{(1-x)} \mathrm{Fe}_{x} \mathrm{O}_{2}$ применена модель прыжковой проводимости, учитывающая влияние тепловых колебаний атомов на резонансный интеграл. Вычислены значения параметра $\varepsilon$ в зависимости от степени легирования $\mathrm{VO}_{2}$.

Работа частично поддержана научной программой ОФН РАН.

DOI: 10.21883/FTT.2018.12.46734.142

\section{1. Введение}

В монокристаллах стехиометрического диоксида ванадия $\left(\mathrm{VO}_{2}\right)$ при температуре $T_{c} \approx 340 \mathrm{~K}$ наблюдается фазовый переход металл-диэлектрик (ПМД). В результате большинство физических свойств этого соединения меняются кардинальным образом $[1,2]$. При температуре выше $340 \mathrm{~K} \mathrm{VO}_{2}$ является металлом (тетрагональная фаза $R)$, а его электропроводность $(\sigma)$ составляет $\sim 10^{4} \Omega^{-1} \cdot \mathrm{cm}^{-1}$ и, как это характерно для металлов, уменьшается с ростом температуры. При охлаждении ниже $T_{c}$ и переходе в моноклинную фазу $M_{1}$ электропроводность диоксида ванадия скачком падает на пять порядков величины и с понижением температуры продолжает уменьшаться. В случае тонких поликристаллических пленок, которые предпочтительны для создания конкретных технических устройств, свойства ПМД в целом сохраняются, однако параметры перехода несколько изменяются. В частности, величина скачка электропроводности уменьшается до трех порядков величины, а сам переход размывается в средней крутой части ПМД на несколько градусов, тогда как на краях перехода это размытие составляет более $10 \mathrm{~K}$. Мощным фактором, влияющим на ПМД в $\mathrm{VO}_{2}$, является легирование. Легирующие примеси являются примесями замещения, и по характеру действия на фазовый переход (ФП) их можно разбить на две группы: группа $\mathrm{Nb}$ и группа $\mathrm{Cr}$. K группе $\mathrm{Nb}$ относятся Mo, W, Re, действие которых сводится, в основном, к снижению температуры ФП. Никаких новых низкотемпературных фаз кроме фазы $M_{1}$ при этом не образуется. Понижение $T_{c}$ при легировании этими примесями связывают с увеличением параметров решетки и с образованием ионов $\mathrm{V}^{3+}$ [3-5]. К группе $\mathrm{Cr}$ относятся $\mathrm{Al}, \mathrm{Fe}, \mathrm{Ga}$. Эти присадки превращают $\mathrm{V}^{4+}$ в решетке в $\mathrm{V}^{5+}$ и способствуют стабилизации двух новых низкотемпературных фаз $-M_{2}$ и $T$, фазовая диаграмма которых получена в [6]. Моноклинная фаза $M_{1}$ может быть представлена как результат двух искажений: спаривания $\mathrm{V}$ атомов вдоль оси $(001)_{R}$ и зигзагообразного искажения вдоль осей кислородного октаэдра. В моноклинной фазе $M_{2}$ половина атомов $\mathrm{V}$ (подрешетка А) спарены и распологаются вдоль оси $(001)_{R}$, а другая половина (подрешетка В) образует зигзагообразные цепочки вдоль того же направления. Триклинная фаза $T$ характеризуется нарастанием наклонов в подрешетке А и увеличением димеризации зигзагообразных цепочек в подрешетке В, что в конце концов приводит к эквивалентным подрешеткам А и В в фазе $M_{1}$. Подчеркнем, что катионы примесей рассматриваются не просто как доноры или акцепторы, но и как центры внутреннего расширения или сжатия кристаллической структуры соответственно, а наличие внутренних или внешних упругих напряжений оказывает сильнейшее влияние на процесс ПМД в диоксиде ванадия [7-9].

Исследованию легированных монокристаллов и тонких пленок диоксида ванадия в последние годы уделяется большое внимание, поскольку это может позволить объяснить природу фазового перехода [10-13]. Интерес к этому соединению связан еще и с развитием нано технологий [7].

Несмотря на то, что $\mathrm{VO}_{2}$ интенсивно исследуется на протяжении нескольких десятилетий, до сих пор не решен вопрос о природе ПМД в этом соединении, как влияют электрон-фононное взаимодействие и электронные корреляции на характеристики этого оксида, а экспериментальные данные по электропроводности чистого и легированного диоксида ванадия фрагментарны 
и проведены в узком температурном диапозоне. Ранее мы исследовали электропроводность чистого диоксида ванадия в широком интервале температур и показали, что электроперенос в $\mathrm{VO}_{2}$ осуществляется прыжками поляронов малого радиуса, на которые оказывают влияние тепловые колебания решетки [1]. В рамках такого подхода в предлагаемой работе мы рассматриваем свойства диоксида ванадия, легированного железом.

\section{2. Эксперимент}

Тонкие пленки диоксида ванадия $\mathrm{V}_{(1-x)} \mathrm{Fe}_{x} \mathrm{O}_{2}$ были синтезированы методом одновременного лазерного напыления из мишеней металлического V (99.9\%) и металлического $\mathrm{Fe}(99.8 \%)$ в атмосфере кислорода при температуре 750-900 К. Так как температуры плавления ванадия и железа близки, о степени легирования $(x)$ судили по относительному времени испарения каждой из мишеней. В качестве подложек использовали сапфир $\left(\mathrm{Al}_{2} \mathrm{O}_{3}\right)$. Толщина пленок составляла 50-60 nm.

За процессом температурного фазового перехода следили по изменению отражательной способности исследуемых пленок на длине волны $\lambda=1.54 \mu \mathrm{m}$ и по изменению электропроводности этих пленок, измеренной по стандартной четырехзондовой методике. В последнем случае перед синтезом пленки на подложку методом лазерного напыления в вакууме наносились платиновые электроды.

\section{3. Результаты и обсуждение}

Температурные зависимости электропроводности тонких пленок чистого и легированного железом диоксида ванадия представлены на рис. 1. Из рисунка видно, что по мере увеличения концентрации железа, температура ПМД смещается в сторону низких температур. При небольших концентрациях примеси параметры ПМД, за исключением $T_{c}$, практически не меняются. Как и в чистом $\mathrm{VO}_{2}$ скачок электропроводности в легированных образцах составляет три порядка величины, а ширина гистерезиса остается равной $8 \mathrm{~K}$. Однако при дальнейшем увеличении концентрации железа ПМД начинает деградировать, что проявляется в значительном уменьшении скачка электропроводности, уширении области перехода, но сама температура ПМД перестает смещаться в сторону низких температур. По-видимому это связано с ослаблением фазового перехода первого рода и постепенного превращения его в переход второго рода из-за размытия фазовых границ диэлектрических фаз $M_{1}$, $M_{2}$ и $T$. Мы не будем обсуждать деградацию ПМД происходящую при высоких концентрациях примеси, а подробно остановимся на области концентраций железа, где параметры ПМД практически не меняются, смещается лишь температура $T_{c}$. На рис. $2, a, b$ соответственно показаны зависимости $\sigma(T)$ в области ПМД при нагреве и охлаждении для чистого $\mathrm{VO}_{2}$ и $\mathrm{V}_{(1-x)} \mathrm{Fe}_{x} \mathrm{O}_{2}(x=0.04$

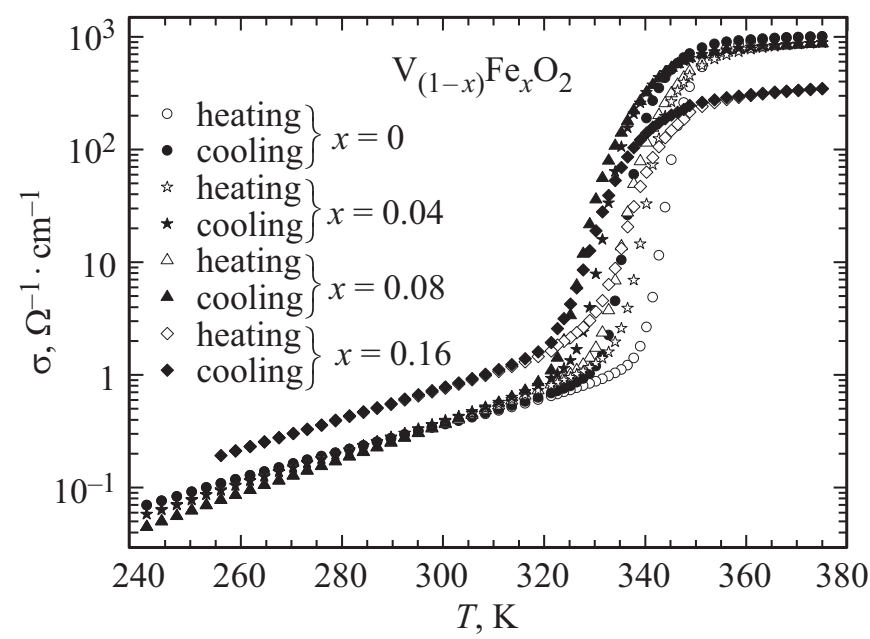

Рис. 1. Температурные зависимости электропроводности пленок $\mathrm{V}_{(1-x)} \mathrm{Fe}_{x} \mathrm{O}_{2}$ при изменении концентрации железа.
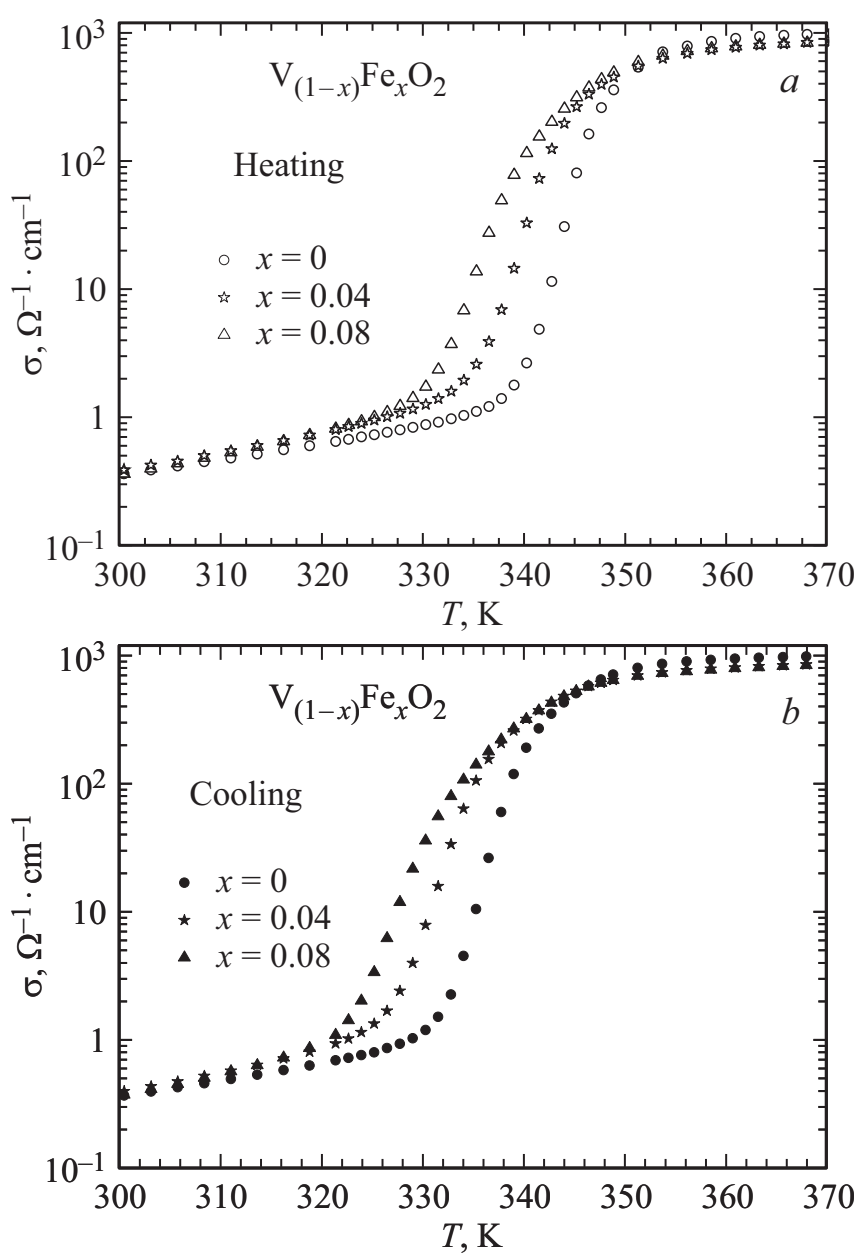

Рис. 2. Температурные зависимости электропроводности пленок чистого и легированного железом $\mathrm{VO}_{2}$ в области фазового перехода: $a-$ полученные при нагревании; $b-$ полученные при охлаждении. 
и 0.08). Отметим, что концентрация примеси в нашем случае значительно выше, чем в [6]. Величина электропроводности в металлической фазе для представленных образцов составляет $\sigma_{m} \sim 10^{3} \Omega^{-1} \cdot \mathrm{cm}^{-1}$, что близко к мотовскому пределу для минимальной металлической проводимости [14], однако характер $\sigma(T)$ не является металлическим. Это, по-видимому, связано с андерсоновской локализацией вызванной неоднородностями состава зерен и дефектами в межзеренных границах (пленки поликристаллические). Заметим, что в монокристаллах $\sigma_{m} \sim 10^{4} \Omega^{-1} \cdot \mathrm{cm}^{-1}$ и проводимость носит металлический характер [1]. Фазовый переход в поликристаллической пленке отличается от монокристалла не только величиной скачка $\sigma$, но и формой температурной зависимости перехода. В монокристалле это - вертикальная линия, обусловленная лавинообразным переходом по всему объему образца. В поликристаллической пленке лавинообразный переход происходит самостоятельно в объеме каждого зерна и поэтому линия $\sigma(T)$ в области ПМД даже в средней, наиболее крутой, части размазана на $\Delta T \sim 8 \mathrm{~K}$. В добавление к этому, в начале и конце перехода имеются „крылья“, растянутые на $\Delta T \sim 20 \mathrm{~K}$. Ширина гистерезиса в наших пленках равна $\Delta T \sim 8 \mathrm{~K}$, а температурный интервал, в котором происходит ПМД, растянут на $\Delta T \sim 50 \mathrm{~K}$ (расстояние между точками, где прямая и обратная ветви гистерезиса сливаются). На уширение области ПМД влияют также эффекты протекания [15]. Поэтому на кривых отражательной способности пленок от температуры, где эффекты протекания отсутствуют, эта область более узкая. Из сравнения формы ветвей гистерезиса при нагреве и охлаждении в чистом и легированном $\mathrm{VO}_{2}$ (рис. 2) следует, что в $\mathrm{V}_{(1-x)} \mathrm{Fe}_{x} \mathrm{O}_{2}$ верхняя треть ветвей гистерезиса избыточно размывается и наклоняются в сторону высоких температур, поглощая часть высокотемпературного „крыла“ гистерезиса. Возможно, такое поведение является следствием расщепления ПМД, связанным с последовательным переходом фазовых границ $R-M_{2}$ и $M_{2}-T$. Из-за „размазки“ по температуре эти переходы сливаются в один, но из сравнения с чистым $\mathrm{VO}_{2}$, где присутствует только одна фазовая граница $R-M_{1}$, подобный механизм кажется разумным. Отметим, что при измерении магнитной восприимчивости в $\mathrm{V}_{(1-x)} \mathrm{Fe}_{x} \mathrm{O}_{2}$, также наблюдался двойной переход [16].

Результаты измерения электропроводности чистого и легированного железом $\mathrm{VO}_{2}$ после перехода в диэлектрическую фазу при охлаждении до температуры $T=240 \mathrm{~K}$ показаны на рис. 1 . Видно, что зависимость $\sigma(T)$ линейна в координатах $\lg (\sigma)$ от $T$. Ранее мы исследовали электропроводность диоксида ванадия и показали, что его проводимость описывается прыжками поляронов малого радиуса, на которые влияют тепловые колебания решетки [1]. Так как механизм переноса заряда в диэлектрической фазе при легировании диоксида ванадия меняться не должен, то уместно рассматривать электропроводность $\mathrm{V}_{(1-x)} \mathrm{Fe}_{x} \mathrm{O}_{2}$ в рамках модели В. Брыксина [17]. В этой модели учитывается эффект теплового смещения атомов решетки на вероятность междоузельных перескоков малых поляронов. Смещения атомов приводят к изменениям в перекрытии волновых функций состояний на соседних узлах. Этим перекрытием определяется резонансный интеграл $(I)$. В первом приближении $I$ меняется с расстоянием $(R)$, на котором происходит перескок, как $\exp (-\alpha R)$, где $\alpha^{-1}$ есть эффективный радиус локализации. В свою очередь, прыжковая подвижность носителя заряда, определяющая электропроводность диоксида ванадия пропорциональна $I^{2}$. Для малых величин $\alpha^{-1}$, порядка амплитуды решеточных колебаний $(\rho)$, можно предположить, что $I^{2}$ должен зависеть линейно от $\rho$. Поэтому в случае зависимости $I^{2}$ от $\rho$ допустимо заменить $I^{2}$ на $\left\langle I^{2}\right\rangle$, где угловые скобки обозначают фононное усреднение через перенормировку фактора Дебая-Уоллера.

$$
\left\langle I^{2}\right\rangle=I^{2} \exp \left(2 \alpha^{2}\left\langle\rho^{2}\right\rangle\right)
$$

где $\left\langle\rho^{2}\right\rangle$ - среднеквадратичное тепловое смещение атомов на узлах решетки. Расчет прыжковой проводимости в модели поляронов малого радиуса с учетом влияния тепловых колебаний решетки на резонансный интеграл приводит к следующей зависимости электропроводности от температуры:

$$
\sigma=e n \frac{e a^{2}}{2 h} \frac{\pi^{1 / 2} I^{2}}{E_{a}^{1 / 2}\left(k_{\mathrm{B}} T\right)^{3 / 2}} \exp \left\{-E_{a} / k_{\mathrm{B}} T+k_{\mathrm{B}} T / \varepsilon\right\}
$$

где $a$ - постоянная решетки, $E_{a}-$ энергия, требуемая для осуществления перескока электрона, $n-$ концентрация носителей заряда, $e-$ заряд электрона, $h$ - постоянная Планка, $\varepsilon-$ величина, имеющая размерность энергии и учитывающая влияние тепловых колебаний решетки на резонансный интеграл. В области высоких температур, когда $2 k_{\mathrm{B}} T>\hbar \omega_{q}$ (где $\hbar=h / 2 \pi$, $\omega_{q}$ - частота оптического фонона), $\varepsilon$ связана со среднеквадратичным тепловым смещением $\left\langle\rho^{2}\right\rangle$ соотношением

$$
\varepsilon=k_{\mathrm{B}} T / 2 \alpha^{2}\left\langle\rho^{2}\right\rangle
$$

Анализ выражения (2) показывает, что при низких температурах второй член под экспонентой становится пренебрежимо малым по сравнению с первым, тогда как при высоких температурах доминирует уже второй член. Поэтому в низкотемпературном пределе выражение (2) может быть представлено в виде

$$
\ln \left(\sigma T^{3 / 2}\right)=A-E_{a} / k_{\mathrm{B}} T
$$

где $A$ и $E_{a}$ не зависят от температуры.

Напротив, в высокотемпературном пределе выражение (2) может быть представлено как:

$$
\ln \left(\sigma T^{3 / 2}\right)=A+k_{\mathrm{B}} T / \varepsilon
$$

где $A$ и $\varepsilon$ не зависят от температуры.

В [1] мы показали, что $\sigma(T) \mathrm{VO}_{2}$ в области температур выше $T \sim 240 \mathrm{~K}$ может быть описана зависимостью (5). Эта зависимость для $\mathrm{VO}_{2}$ и $\mathrm{V}_{(1-x)} \mathrm{Fe}_{x} \mathrm{O}_{2}$ 


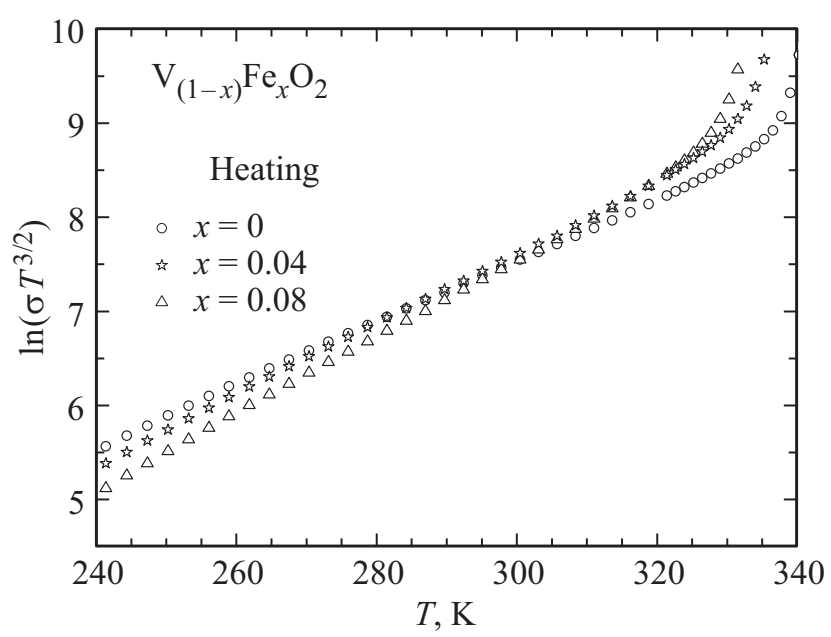

Рис. 3. Зависимость $\ln \left(\sigma T^{3 / 2}\right)=A+k_{\mathrm{B}} T / \varepsilon \quad$ пленок $\mathrm{V}_{(1-x)} \mathrm{Fe}_{x} \mathrm{O}_{2}$ с разной концентрацией железа.

$(x=0.04$ и 0.08$)$ представлена на рис. 3. Видно, что при повышении концентрации железа наклон прямой, равный $\varepsilon^{-1}$, увеличивается. Значения $\varepsilon$ для $\mathrm{VO}_{2}$ и $\mathrm{V}_{(1-x)} \mathrm{Fe}_{x} \mathrm{O}_{2} \quad(x=0.04$ и 0.08$)$ получились равными $2.72 \cdot 10^{-3} \mathrm{eV}, 2.35 \cdot 10^{-3} \mathrm{eV}$ и $2.15 \cdot 10^{-3} \mathrm{eV}$, соответственно. Величина $\varepsilon^{-1}$ пропорциональна времени туннелирования малого полярона через барьер между соседними узлами. Иными словами подвижность полярона тем больше, чем меньше величина $\varepsilon^{-1}$. Из наших данных следует, что по мере увеличения концентрации железа в диоксиде ванадия увеличивается и величина $\varepsilon^{-1}$, а это можно трактовать как усиление локализации носителя заряда на узле вследствие расширения кристаллической решетки, что приводит к понижению температуры ПМД.

\section{4. Заключение}

Показано, что легирование железом диоксида ванадия приводит к существенному изменению температурной зависимости электропроводности $\mathrm{V}_{(1-x)} \mathrm{Fe}_{x} \mathrm{O}_{2}$ по сравнению с чистым $\mathrm{VO}_{2}$. Показано, что дополнительное размытие $\sigma(T)$ в области ПМД при приближении к металлической фазе в легированных образцах $\mathrm{VO}_{2}$, может быть следствием образования дополнительных фазовых границ $R-M_{2}$ и $M_{2}-T$. Электропроводность диэлектрической фазы $\mathrm{V}_{(1-x)} \mathrm{Fe}_{x} \mathrm{O}_{2}$ хорошо описывается моделью малого полярона, учитывающей влияние тепловых колебаний атомов решетки на резонансный интеграл. Определен характерный параметр модели $\varepsilon$ для чистого и легированного $\mathrm{VO}_{2}$. Показано, что энергия $\varepsilon$, учитывающая влияние тепловых колебаний решетки на вероятность туннелирования малого полярона через барьер между соседними узлами, уменьшается с ростом концентрации легирующей примеси. Возможно это связано с увеличением параметров решетки.

\section{Список литературы}

[1] В.Н. Андреев, В.А. Климов. ФТТ 49, 2146 (2007).

[2] A. Zilbersztejn, N.F. Mott. Phys. Rev. B 11, 4383 (1975).

[3] В.Н. Андреев, В.А. Климов. ФТТ 58, 590 (2016).

[4] G. Villeneuve, A. Bordet, A. Casalot, J.P. Pouget, H. Launois, P. Lederer. J. Phys. Chem. Solids 33, 1953 (1972).

[5] M. Ritschel, N. Mattern, W. Bruckner, H. Oppermann, G. Strover, W. Moldenhauer, J. Henre, E. Wolf. Krist. Techn. 12, 1221 (1977).

[6] M. Marezio, D.B. McWhan, J.P. Remeika, P.D. Dernier. Phys. Rev. B 7, 2541 (1972).

[7] J.H. Park, J.M. Coy, T.S. Kasirga, C. Huang, Z. Fei, S. Hunter, D.H. Gobden. Nature 500, 431 (2013).

[8] J. Wu, Q. Gu, B.S. Guiton, N.P. de Leon, L. Ouyang, H. Park. Nano Lett. 6, 2313 (2006).

[9] A. Tselev, I.A. Luk'yanchuk, I.N. Ivanov, J.D. Budai, J.Z. Tischler, E. Strelkov, K. Jones, A. Kolmakov, S.V. Kalinin. Nano Lett. 10, 4409 (2010).

[10] E. Strelkov, A. Tselev, I. Ivanov, J.D. Budai, J. Zhang, J.Z. Tischler, I. Kravchenko, S.V. Kalinin, A. Kolmakov. Nano Lett. 12, 6198 (2012).

[11] T.J. Hanlon, J.A. Coath, M.A. Richardson. Thin Solid Films 436, 269 (2003).

[12] C. Marini, E. Arcangeletti, D.Di Castro, L. Baldassare, A. Perucchi, S. Lupi, L. Malavasi, L. Boeri, E. Pomjakushina, K. Conder, P. Postorino. Phys. Rev. B 77, 235111 (2008).

[13] В.Н. Андреев, В.А. Климов, М.Е. Компан, Б.А. Мелех. ФТТ 56, 1802 (2014)

[14] N.F. Mott. Metall-Insulator Transitions. Tailor and Francis, LTD, London (1974).

[15] V.N. Andreev, T.V. Smirnova, F.A. Chudnovskii. Phys. Status Solidi B 77, K97 (1977).

[16] E. Pollert, G. Villeneuve, F. Menil, P. Hagenmuller. Mater. Res. Bull. 11, 159 (1976).

[17] В.В. Брыксин. ЖЭТФ 100, 1556 (1991).

Редактор К.В. Емцев 